\title{
Do we always consistently define the clinically important echocardiographic parameters?
}

\author{
Jaroslav Meluzina, , Zdenka Gregorovaa, , Helena Podrouzkovaa, , Roman Panovskya,
}

\begin{abstract}
Aims. The aim of this short communication is to highlight some inconsistencies in defining two clinically important echocardiographic parameters with possible impact on clinical practice.

Methods and Results. A surview of publications in reputable cardiological journals revealed inconsistencies posing questions on the validity of the published data and on the scrupulousness of the reviewing process. We demonstrate examples of inconsistencies in the calculation of left ventricular mass using Devereux's formula and in the definition of relative wall thickness, two echocardiographic parameters commonly utilized in a clinical practice.
\end{abstract}

Conclusion. It is desirable to follow exactly the definitions of more complex parameters and to unify ways of presenting those with several definitions.

Key words: left ventricular mass, relative wall thickness

Received: January 5, 2012; Accepted: May 22, 2012; Available online: June 14, 2012

http://dx.doi.org/10.5507/bp.2012.058

${ }^{a}$ 1st Department of Internal Medicine - Cardioangiology, Faculty of Medicine, Masaryk University Brno, Czech Republic

${ }^{b}$ Department of Cardiology, St. Anne's Hospital, ICRC, Brno

Corresponding author: Jaroslav Meluzin, e-mail: jaroslav.meluzin@fnusa.cz

\section{INTRODUCTION}

In medical practice, it is of the utmost importance to follow strictly definitions, formulas, and units defining exactly various parameters, pathological situations, and diseases, with the aim of distinguishing normal versus pathological conditions. In this regard, mistakes and inconsistencies may have disastrous consequences for our patients because they can influence negatively diagnostic processes and treatment strategies. The purpose of this short communication is to demonstrate some inconsistencies and mistakes encountered even in very reputable cardiological journals and to warn against all such phenomena.

\section{METHODS AND RESULTS}

Examples of inconsistently defined baseline echocardiographic parameters in the literature

In this paper, we would like to demonstrate confusing definitions and formulas concerning two frequently used echocardiographic parameters, left ventricular (LV) mass and relative wall thickness. Both parameters are frequently clinically utilized in numerous clinical situations such as the diagnostics of heart failure with normal ejection fraction, the assessment of LV remodelling process under various pathological situations, determination of the hypertensive heart disease, etc.
Echocardiographic assessment of left ventricular mass index

LV hypertrophy is an important mechanism of myocardial adaptation to hemodynamic overload on the heart or contractile myocardial failure. In a clinical practice, LV hypertrophy is defined and quantified by calculating LV mass using most frequently Devereux's formula ${ }^{1}$. It is derived from the American Society of Echocardiography (ASE) measurements incorporated into the fundamental equation ${ }^{1}$ (ASE-cube LV mass): ASE-cube LV mass = 1.04 [(IVSd + LVIDd + PWTd $)^{3}-$ LVIDd $\left.^{3}\right\}$, where IVSd = interventricular septal thickness, LVIDd $=\mathrm{LV}$ internal diameter, and PWTd = posterior wall thickness, all measured at end-diastole (d). However, when confronted with anatomic LV mass measurements made at necropsy, the ASE-cube method overestimated LV mass significantly and required correction. As demonstrated by Devereux et al. ${ }^{1}$, the optimal correction was achieved by the following regression equation: $\mathrm{LV}$ mass $=0.8$ (ASE-cube $\mathrm{LV}$ mass $)+0.6 \mathrm{~g}$. Thus, the resultant Devereux's formula for calculating LV mass is as follows: LV mass $=0.8[1.04$ $\left[(\text { IVSd + LVIDd + PWTd })^{3}-\right.$ LVIDd $\left.\left.^{3}\right\}\right]+0.6$ g. The same formula is included in the fundamental echocardiographic document such as ASE/EAE (European Association of Echocardiography) recommendations for chamber quantification ${ }^{2}$ and, in our opinion, represents the true Devereux's formula.

However, the surprisingly large amount of reports published in many reputable journals used the distinct "Devereux's formula"": LV mass $=0.8$ [1.04 $(\mathrm{IVSd}+$ LVIDd + PWTd $\left.)^{3}-(\text { LVIDd })^{3}\right]+0.6 \mathrm{~g}\left(\right.$ ref. $\left.^{3-8}\right)$. Even if the change in formula is „small“ (different position of 
parentheses), the results derived from such a "modified" equation are different as compared to the original formula. For example, patient with septal and posterior wall thicknesses of $15 \mathrm{~mm}$ and LVIDd of $60 \mathrm{~mm}$ has LV mass $427.4 \mathrm{~g}$ when using the correct Devereux's formula and $434.3 \mathrm{~g}$ when using "modified" Devereux's formula. It results in the problem with the comparison of data from various studies, because the majority of reports evaluating the LV mass does not include into their methodology the Devereux's formula, but uses only the reference on the Devereux's paper. In such cases, it is unclear whether LV mass is derived from the correct formula. One can only speculate on the reasons for these discrepancies in the definition of LV mass. We suppose that they are likely due to authors' inattention (the wrong formula may be rewritten automatically from the previous papers) and the careless reviewing process.

\section{Evaluation of the relative wall thickness}

The relative wall thickness (RWT) is an important parameter that helps to categorize remodelling process into the concentric remodelling (increase in RWT, normal LV mass), concentric hypertrophy (increase in RWT, increase in LV mass), eccentric hypertrophy (normal RWT, increase in LV mass), and normal geometry (normal RWT and LV mass). The basic document, guidelines of the ASE and EAE summarized in the "Recommendations for chamber quantification" ( ref. $\left.^{2}\right)$ defines the RWT by the formula: $\mathrm{RWT}=(2 \times \mathrm{PWTd}) /$ LVIDd. Some papers follow this definition ${ }^{9,10}$. Hovewer, in other papers ${ }^{5,11,12}$, the different formula is used: RWT $=($ IVSd + PWTd $) /$ LVIDd. The latter formula provides markedly different results mainly in patients with hypertrophic cardiomyopathy having asymetrical septal hypertrophy. Thus, without the knowledge of the original formula for RWT calculation, the comparison of results from various papers may be confusing and may lead to wrong conclusions.

\section{CONCLUSION}

It is desirable to follow exactly the definitions of more complex parameters and to unify the way of presenting those with more potential definitions.

\section{ABBREVIATIONS}

ASE, American Society of Echocardiography; EAE, European Association of Echocardiography; IVSd, Interventricular septal thickness; LV, Left ventricular; LVIDd, Left ventricular internal diameter; PWTd, Posterior wall thickness; RWT, Relative wall thickness.

\section{ACKNOWLEDGEMENT}

The study was supported by the European Regional Development Fund - Project FNUSA-ICRC (No. CZ.1.05/1.1.00/02.0123).

\section{CONFLICT OF INTEREST STATEMENT}

The authors state that there are no conflicts of interest regarding the publication of this article.

\section{REFERENCES}

1. Devereux RB, Alonso DR, Lutas EM, Gottlieb GJ, Campo E, Sachs I, and Reichek N. Echocardiographic assessment of left ventricular hypertrophy: comparison to necropsy findings. Am J Cardiol 1986;57:4508.

2. Lang RM, Bierig M, Devereux RB, Flachskampf FA, Foster E, Pellikka P, Picard MH, Roman MJ, Seward J, Shanewise J, Solomon S, Spencer KT, Sutton MSJ, Stewart W. Recommendations for chamber quantification. Eur J Echocardiogr 2006;7:79-108.

3. Shin SH, Park MY, Oh WJ, Hong SJ, Pak HN, Song WH, Lim DS, Kim $\mathrm{YH}$, and Shim WJ. Left atrial volume is a predictor of atrial fibrillation recurrence after catheter ablation. J Am Soc Echocardiogr 2008:21:697-702.

4. Sundström J, Sullivan L, Selhub J, Benjamin EJ, D'Agostino RB, Jacques PF, Rosenberg IH, Levy D, Wilson PWF, Vasan RS. Relation of plasma homocysteine to left ventricular structure and function: the Framingham Heart Study. Europ Heart J 2004;25:523-30.

5. Sundström J, Evans JC, Benjamin EJ, Levy D, Larson MG, Sawyer DB, Siwik DA, Colucci WS, Wilson PWF, Vasan RS. Relations of plasma total TIMP-1 levels to cardiovascular risk factors and echocardiographic measures: the Framingham Heart Study. Europ Heart J 2004;25:1509-16.

6. Wang TJ, Larson MG, Benjamin EJ, Siwik DA, Safa R, Guo ChY, Corey D, Sundstrom J, Sawyer DB, Colucci WS, and Vasan RS. Clinical and echocardiographic correlates of plasma procollagen type III aminoterminal peptide levels in the community. Am Heart J 2007;154:2917.

7. Dhingra R, Pencina MJ, Schrader P, Wang TJ, Levy D, Pencina K, Siwik DA, Colucci WS, Benjamin EJ, Vasan RS. Relations of matrix remodeling biomarkers to blood pressure progression and incidence of hypertension in the community. Circulation 2009;119:1101-7.

8. Kücherer HF and Kuebler WW. Diagnosis of left ventricular hypertrophy by echocardiography. J Cardiovas Pharmacol 1992;19(Suppl.5):S81-S86.

9. Kosmala W, Plaksej R, Strotmann JM, Weigel C, Herrmann S, Niemann M, Mende H, Störk S, Angermann ChE, Wagner JA, and Weidemann F. Progression of left ventricular functional abnormalities in hypertensive patients with heart failure: an ultrasonic two-dimensional speckle tracking study. J Am Soc Echocardiogr 2008;21:1309-17.

10. Pavlopoulos H, Grapsa J, Stefanadi E, Philippou E, Dawson D, Nihoyannopoulos P. Is it only diastolic dysfunction? Segmental relaxation patterns and longitudinal systolic deformation in systemic hypertension. Eur J Echocardiogr 2008;9:741-7.

11. Kansal MM, Lester SJ, Surapaneni P, Sengupta PP, Appleton ChP, Ommen SR, Ressler SW, and Hurst RT. Usefulness of two-dimensional and speckle tracking echocardiography in "gray zone" left ventricular hypertrophy to differentiate professional football player's heart from hypertrophic cardiomyopathy. Am J Cardiol 2011;108:1322-6.

12. Chahal NS, Lim TK, Jain P, Chambers JC, Kooner JS, and Senior R. New insights into the relationship of left ventricular geometry and left ventricular mass with cardiac function: a population study of hypertensive subjects. Europ Heart J 2010;31:588-94. 